\title{
Forces within hadrons on the light front
}

\author{
Adam Freese $\odot^{*}$ and Gerald A. Miller $\odot^{\dagger}$ \\ Department of Physics, University of Washington, Seattle, Washington 98195, USA
}

(Received 25 February 2021; accepted 21 April 2021; published 18 May 2021)

\begin{abstract}
In this work, we find the light front densities for momentum and forces, including pressure and shear forces, within hadrons. This is achieved by deriving relativistically correct expressions relating these densities to the gravitational form factors $A(t)$ and $D(t)$ associated with the energy momentum tensor. The derivation begins from the fundamental definition of density in a quantum field theory, namely the expectation value of a local operator within a spatially localized state. We find that it is necessary to use the light front formalism to define a density that corresponds to the internal hadron structure. When using the instant form formalism, it is impossible to remove the spatial extent of the hadron wave function from any density, and —even within instant form dynamics—one does not obtain a Breit frame Fourier transform for a properly defined density. Within the front formalism, we derive new expressions for various mechanical properties of hadrons, including the mechanical radius, as well as for stability conditions. The multipole ansatz for the form factors is used as an example to illustrate all of these findings.
\end{abstract}

DOI: 10.1103/PhysRevD.103.094023

\section{INTRODUCTION}

In the past few years, the energy momentum tensor (EMT) has become a major focus of both theoretical and experimental efforts in hadron physics. The proton mass puzzle [1-6] and proton spin puzzle [7-9] are major motivators for this focus. Matrix elements of the EMT between plane wave states define gravitational form factors [10], which provide information about both the quarkgluon decomposition and the spatial distribution of energy, momentum, and angular momentum. These form factors are in principle accessible through high-energy reactions such as deeply virtual Compton scattering [11-13] at facilities such as Jefferson Lab [14-16] and the upcoming Electron Ion Collider [17], as well as in $\gamma \gamma^{*} \rightarrow$ hadrons at Belle [18].

Investigations into both the EMT and into deeply virtual Compton scattering have led to the discovery [19] of an additional form factor $D(t)$, called the " $D$-term" or "Druckterm" [20], which does not encode a conserved current, but instead contains information about the spatial distribution of forces within the hadron [21,22]. The $D$-term has attracted a lot of interest lately [22-29], and remains largely experimentally unexplored.

\footnotetext{
afreese@uw.edu

†miller@uw.edu
}

Published by the American Physical Society under the terms of the Creative Commons Attribution 4.0 International license. Further distribution of this work must maintain attribution to the author(s) and the published article's title, journal citation, and DOI. Funded by SCOAP ${ }^{3}$.
Since EMT form factors such as the $D$-term encode spatial densities within hadrons, it is important that the relationship between the form factors and spatial densities is properly derived and understood. The connection between form factors and spatial densities has been presented in terms of Fourier transforms [22,30-32]. Improvements [31-35] in the literature have been made since the original work [30] that used three-dimensional form factors. Specifically, three-dimensional Fourier transforms at fixed (instant form) time have been shown to be incorrect [35] because of the impossibility of localizing the center of momentum in three dimensions [36]. Twodimensional Fourier transforms at fixed light front time have been used as a correct alternative $[31,33,34]$.

The improvements have been applied only to electromagnetic form factors and the associated charge densities, but the same concerns are also present for the EMT form factors and energy, spin, and force densities. It is thus prudent to consider carefully how to properly associate spatial densities in hadrons with the EMT. In this paper, we derive-rather than postulate-a connection between the EMT form factors on the one hand and spatial densities of momentum and forces on the other. In particular, we derive the two-dimensional light front Fourier transform as providing this connection.

This work is organized as follows. In Sec. II, we derive the connection between spatial densities associated with an arbitrary local operator and its matrix elements between plane wave states. This is done both at fixed (instant form) time and at fixed light front time. We find only the latter can define a density that depends only on internal hadron structure, rather than on irremovable wave function spread. 
In Sec. III, we analyze the classical continuum EMT on the light front in order to obtain associations between the EMT form factors and specific properties of hadrons (specifically stresses). In Sec. IV, we combine the results of the previous sections to obtain results for the light front momentum density and the light front stress tensor. Then, in Sec. V, we illustrate our results with a few model examples, and we conclude and provide an outlook in Sec. VI.

\section{SPATIAL DENSITIES AND FOURIER TRANSFORMS}

Spatial densities in quantum field theories are given by expectation values of local operators with physically realizable states. Given a local operator $\hat{\mathcal{O}}(x)$ — such as the electromagnetic current or the energy-momentum tensor - and a physically realizable one-hadron state $|\Psi\rangle$, the relevant density is given by $\langle\Psi|\hat{\mathcal{O}}(x)| \Psi\rangle$.

Form factors are also associated with the local operator $\hat{\mathcal{O}}(x)$ through the matrix element between distinct oneparticle plane wave states, namely through $\left\langle p^{\prime}, \lambda^{\prime}\right| \hat{\mathcal{O}}(0)$ $|p, \lambda\rangle$. It is common in the hadron physics literature to associate the plane wave matrix element with spatial densities using a Fourier transform, with spacelike components of $\Delta=p^{\prime}-p$ as the conjugate momentum variable [22,30-32]. In particular, two varieties of Fourier transform exist in the literature: a three-dimensional "Breit frame" Fourier transform at fixed instant form time $x^{0}=0$ (see, e.g., Refs. [22,30,32]), and a two-dimensional Fourier transform over transverse coordinates at fixed light front time $x^{+}=0$ (see, e.g., Refs. [31,33,34]). The validity of the Breit frame transform as a true spatial density has been called into question [35,36].

In this section, we shall derive the correct association between the matrix elements $\left\langle p^{\prime}, \lambda^{\prime}|\hat{\mathcal{O}}(0)| p, \lambda\right\rangle$ and the actual field-theoretic spatial densities $\langle\Psi|\hat{\mathcal{O}}(x)| \Psi\rangle$ for one-hadron states, using both fixed light front time and fixed instant form time. We will discuss under what conditions the former can be simplified to an ordinary twodimensional Fourier transform over transverse momentum transfer, and will demonstrate that the latter does not become a "Breit frame" Fourier transform.

\section{A. Using the front form}

We shall begin with the light front case. The light front coordinates are defined so that $x^{ \pm}=\frac{1}{\sqrt{2}}\left(x^{0} \pm x^{3}\right)$. The density is defined at fixed light front time $x^{+}=0$, and the null spatial coordinate $x^{-}$will be integrated out, leaving a two-dimensional spatial density over transverse coordinates $\mathbf{x}_{\perp}$. To start, the following completeness relation for one-hadron states:

$$
\sum_{\lambda} \int \frac{\mathrm{d} p^{+} \mathrm{d}^{2} \mathbf{p}_{\perp}}{2 p^{+}(2 \pi)^{3}}\left|p^{+}, \mathbf{p}, \lambda\right\rangle\left\langle p^{+}, \mathbf{p}_{\perp}, \lambda\right|=1
$$

is inserted twice into $\langle\Psi|\hat{\mathcal{O}}(x)| \Psi\rangle$, giving

$$
\langle\Psi|\hat{\mathcal{O}}(x)| \Psi\rangle=\sum_{\lambda \lambda^{\prime}} \int \frac{\mathrm{d} p^{+} \mathrm{d}^{2} \mathbf{p}_{\perp}}{2 p^{+}(2 \pi)^{3}} \int \frac{\mathrm{d} p^{\prime+} \mathrm{d}^{2} \mathbf{p}_{\perp}^{\prime}}{2 p^{\prime+}(2 \pi)^{3}}\left\langle\Psi \mid p^{\prime+}, \mathbf{p}_{\perp}^{\prime}, \lambda^{\prime}\right\rangle\left\langle p^{\prime+}, \mathbf{p}_{\perp}^{\prime}, \lambda^{\prime}|\hat{\mathcal{O}}(x)| p^{+}, \mathbf{p}_{\perp}, \lambda\right\rangle\left\langle p^{+}, \mathbf{p}_{\perp}, \lambda \mid \Psi\right\rangle
$$

The spacetime dependence of the local operator $\hat{\mathcal{O}}(x)$ is given by $\hat{\mathcal{O}}(x)=e^{i \hat{P} \cdot x} \hat{\mathcal{O}}(0) e^{-i \hat{P} \cdot x}$, and the plane wave states surrounding $\hat{\mathcal{O}}(x)$ are eigenstates of $\hat{P}$. Using light front coordinates at fixed $x^{+}=0$ gives $\hat{P} \cdot x=\hat{P}^{+} x^{-}-\hat{\mathbf{P}}_{\perp} \cdot \mathbf{x}_{\perp}$. If we define a change of variables $P=\frac{1}{2}\left(p+p^{\prime}\right)$ and $\Delta=p^{\prime}-p$, then we find that

$$
\begin{aligned}
\left\langle\Psi\left|\hat{\mathcal{O}}\left(x^{+}=0, x^{-}, \mathbf{x}_{\perp}\right)\right| \Psi\right\rangle= & \sum_{\lambda \lambda^{\prime}} \int \frac{\mathrm{d} P^{+} \mathrm{d}^{2} \mathbf{P}_{\perp}}{2(2 \pi)^{3}} \int \frac{\mathrm{d} \Delta^{+} \mathrm{d}^{2} \boldsymbol{\Delta}_{\perp}}{2(2 \pi)^{3}} \frac{1}{P^{+}+\frac{\Delta^{+}}{2}} \frac{1}{P^{+}-\frac{\Delta^{+}}{2}} \\
& \times\left\langle\Psi \mid p^{\prime+}, \mathbf{p}_{\perp}^{\prime}, \lambda^{\prime}\right\rangle\left\langle p^{\prime+}, \mathbf{p}_{\perp}^{\prime}, \lambda^{\prime}|\hat{\mathcal{O}}(0)| p^{+}, \mathbf{p}_{\perp}, \lambda\right\rangle\left\langle p^{+}, \mathbf{p}_{\perp}, \lambda \mid \Psi\right\rangle e^{i\left(\Delta^{+} x^{-}-\mathbf{\Delta}_{\perp} \cdot \mathbf{x}_{\perp}\right)} .
\end{aligned}
$$

In order to remove the dependence of the density on the state that the hadron is prepared in, it must be possible to factorize the $P$ and $\Delta$ dependence of the integrand. The factors $\left(P^{+}+\frac{\Delta^{+}}{2}\right)^{-1}$ and $\left(P^{+}-\frac{\Delta^{+}}{2}\right)^{-1}$ impede this factorization unless $\Delta^{+}=0$, which can be achieved by integrating out $x^{-}$. Doing so gives us

$$
\begin{aligned}
\int \mathrm{d} x^{-}\left\langle\Psi\left|\hat{\mathcal{O}}\left(x^{+}=0, x^{-}, \mathbf{x}_{\perp}\right)\right| \Psi\right\rangle= & \sum_{\lambda \lambda^{\prime}} \int \frac{\mathrm{d} P^{+} \mathrm{d}^{2} \mathbf{P}_{\perp}}{2 P^{+}(2 \pi)^{3}} \int \frac{\mathrm{d}^{2} \boldsymbol{\Delta}_{\perp}}{(2 \pi)^{2}} \\
& \times\left\langle\Psi \mid P^{+}, \mathbf{p}_{\perp}^{\prime}, \lambda^{\prime}\right\rangle \frac{\left\langle P^{+}, \mathbf{p}_{\perp}^{\prime}, \lambda^{\prime}|\hat{\mathcal{O}}(0)| P^{+}, \mathbf{p}_{\perp}, \lambda\right\rangle}{2 P^{+}}\left\langle P^{+}, \mathbf{p}_{\perp}, \lambda \mid \Psi\right\rangle e^{-i \Delta_{\perp} \cdot \mathbf{x}_{\perp}}
\end{aligned}
$$

This is the general expression for an arbitrary hadron state $|\Psi\rangle$. For such a general state, the density associated with $\hat{\mathcal{O}}(x)$ depends not only on the hadron's internal structure but also on its wave function spread. 
To obtain a density depending only on internal structure, a wave function that is completely localized at the origin should be used. This must be done carefully, since absolute position states do not exist in Hilbert space [37]. In particular, localization is achieved by considering wave packets with arbitrarily narrow width in the position representation, though this width must be kept finite until after all other calculations have been performed [35]. A specific example — which is also a $P^{+}$plane wave state, and a state with definite light front helicity $\Lambda$-is given by

$$
\left\langle p^{+}, \mathbf{p}_{\perp}, \lambda \mid \Psi\right\rangle=\sqrt{2 \pi}(2 \sigma) e^{-\sigma^{2} \mathbf{p}_{\perp}^{2}} \sqrt{2 p^{+}(2 \pi) \delta_{\epsilon}\left(p^{+}-P^{+}\right)} \delta_{\lambda \Lambda},
$$

where $\delta_{\epsilon}(x)$ is an arbitrary representation of the Dirac delta function [such that $\delta_{\epsilon}(x) \rightarrow \delta(x)$ as $\epsilon \rightarrow 0$ ]. Ultimately, the $\sigma \rightarrow 0$ limit should not be taken until after all momentum integrals have been performed, except in special cases such as when the dominated convergence theorem allows the limit to be commuted with integration.

Using the wave function of Eq. (5) in Eq. (4) gives

$$
\begin{aligned}
& \int \mathrm{d} x^{-}\left\langle\Psi\left|\hat{\mathcal{O}}\left(x^{+}=0, x^{-}, \mathbf{x}_{\perp}\right)\right| \Psi\right\rangle \\
& =(2 \pi)(2 \sigma)^{2} \int \frac{\mathrm{d}^{2} \mathbf{P}_{\perp}}{(2 \pi)^{2}} e^{-2 \sigma^{2} \mathbf{P}_{\perp}^{2}} \\
& \quad \times \int \frac{\mathrm{d}^{2} \boldsymbol{\Delta}_{\perp}}{(2 \pi)^{2}} \frac{\left\langle P^{+}, \mathbf{p}_{\perp}^{\prime}, \Lambda|\hat{\mathcal{O}}(0)| P^{+}, \mathbf{p}_{\perp}, \Lambda\right\rangle}{2 P^{+}} e^{-i \Delta_{\perp} \cdot \mathbf{x}_{\perp}} e^{-\frac{\sigma^{2}}{2} \Delta_{\perp}^{2}} .
\end{aligned}
$$

This is the most general possible result for the $x^{-}$-integrated density at $x^{+}=0$. Whether it is possible to proceed from here depends on the form of the specific matrix element $\left\langle P^{+}, \mathbf{p}_{\perp}^{\prime}, \lambda^{\prime}|\hat{\mathcal{O}}(0)| P^{+}, \mathbf{p}_{\perp}, \lambda\right\rangle$. In particular, the possibility of proceeding depends on whether the Lorentz structures in these matrix elements contain any factors of $\mathbf{P}_{\perp}$. If there are no such factors, it is possible to do the $\mathbf{P}_{\perp}$ integration, giving

$$
\begin{aligned}
& \int \mathrm{d} x^{-}\left\langle\Psi\left|\hat{\mathcal{O}}\left(x^{+}=0, x^{-}, \mathbf{x}_{\perp}\right)\right| \Psi\right\rangle \\
& =\int \frac{\mathrm{d}^{2} \boldsymbol{\Delta}_{\perp}}{(2 \pi)^{2}} \frac{\left\langle P^{+}, \mathbf{p}_{\perp}^{\prime}, \Lambda|\hat{\mathcal{O}}(0)| P^{+}, \mathbf{p}_{\perp}, \Lambda\right\rangle}{2 P^{+}} e^{-i \Delta_{\perp} \cdot \mathbf{x}_{\perp}} e^{-\frac{\sigma^{2}}{2} \Delta_{\perp}^{2}},
\end{aligned}
$$

which is the first major result of this section.

On the other hand, if there are additional factors of $\mathbf{P}_{\perp}$, the resulting integral will contain a factor $\sigma^{-1}$ or worse, causing the result to diverge in the $\sigma \rightarrow 0$ limit. This essentially occurs because of complementarity: an observable that depends on $\mathbf{P}_{\perp}$ cannot be well-defined in the limit of the hadron's transverse position being arbitrarily well-known.

It is, of course, possible to calculate finite spatial densities for any current, provided we avoid the limit of arbitrary spatial localization. However, if we wish for our densities to encode the internal structure of hadrons rather than wave function spread, we are constrained to calculating spatial densities for currents that do not depend on $\mathbf{P}_{\perp}$. As we shall see in Sec. III, the spatial components of the energy momentum tensor are not suitable candidates for defining such an internal spatial density. However, we will also find that matrix elements of the pure stress tensorthat is, the local values of the stress tensor in a comoving frame, which encodes pressure and shear-do not depend on $\mathbf{P}_{\perp}$, and therefore the stress tensor is a suitable candidate for internal spatial densities.

\section{B. Using the instant form}

Let us next briefly consider spatial densities at a fixed instant form time $x^{0}=0$. This time we use the completeness relation

$$
\sum_{\lambda} \int \frac{\mathrm{d}^{3} \mathbf{p}}{2 E_{p}(2 \pi)^{3}}|\mathbf{p}, \lambda\rangle\langle\mathbf{p}, \lambda|=1,
$$

inserted twice into the expectation value $\langle\Psi|\hat{\mathcal{O}}(x)| \Psi\rangle$ to find

$$
\langle\Psi|\hat{\mathcal{O}}(x)| \Psi\rangle=\sum_{\lambda \lambda^{\prime}} \int \frac{\mathrm{d}^{3} \mathbf{p}}{2 E_{p}(2 \pi)^{3}} \int \frac{\mathrm{d}^{3} \mathbf{p}^{\prime}}{2 E_{p}^{\prime}(2 \pi)^{3}}\left\langle\Psi \mid \mathbf{p}^{\prime}, \lambda^{\prime}\right\rangle\left\langle\mathbf{p}^{\prime}, \lambda^{\prime}|\hat{\mathcal{O}}(x)| \mathbf{p}, \lambda\right\rangle\langle\mathbf{p}, \lambda \mid \Psi\rangle .
$$

Using the change of variables $\mathbf{P}=\frac{1}{2}\left(\mathbf{p}+\mathbf{p}^{\prime}\right)$ and $\boldsymbol{\Delta}=\mathbf{p}^{\prime}-\mathbf{p}$, and the spatial dependence $\hat{\mathcal{O}}(x)=e^{i \hat{P} \cdot x} \hat{\mathcal{O}}(0) e^{-i \hat{P} \cdot x}$, at fixed time $x^{0}=0$, we find

$$
\left\langle\Psi\left|\hat{\mathcal{O}}\left(\mathbf{x}, x^{0}=0\right)\right| \Psi\right\rangle=\sum_{\lambda \lambda^{\prime}} \int \frac{\mathrm{d}^{3} \mathbf{P}}{(2 \pi)^{3}} \int \frac{\mathrm{d}^{3} \boldsymbol{\Delta}}{(2 \pi)^{3}} \frac{1}{4 E_{p} E_{p}^{\prime}}\left\langle\Psi \mid \mathbf{p}^{\prime}, \lambda^{\prime}\right\rangle\left\langle\mathbf{p}^{\prime}, \lambda^{\prime}|\hat{\mathcal{O}}(0)| \mathbf{p}, \lambda\right\rangle\langle\mathbf{p}, \lambda \mid \Psi\rangle e^{-i \Delta \cdot \mathbf{x}} .
$$

It is controversial whether spatial localization at fixed $x^{0}$ is possible, and proposed localization methods typically redefine the concept of localization to admit some wave function spread [38-40]. As a loose localization procedure in momentum space, we can use the following wave function [36]: 


$$
\langle\mathbf{p}, \lambda \mid \Psi\rangle=\sqrt{2 E_{p}}(2 \pi)^{3 / 4}(2 \sigma)^{3 / 2} e^{-\sigma^{2} \mathbf{p}^{2}} \delta_{\lambda \Lambda}
$$

Using this wave function, we find

$$
\left\langle\Psi\left|\hat{\mathcal{O}}\left(\mathbf{x}, x^{0}=0\right)\right| \Psi\right\rangle=(2 \pi)^{3 / 2}(2 \sigma)^{3} \int \frac{\mathrm{d}^{3} \mathbf{P}}{(2 \pi)^{3}} \int \frac{\mathrm{d}^{3} \Delta}{(2 \pi)^{3}} \sqrt{\frac{1}{4 E_{p} E_{p}^{\prime}}} e^{-2 \sigma^{2} \mathbf{P}^{2}}\left\langle\mathbf{p}^{\prime}, \Lambda|\hat{\mathcal{O}}(0)| \mathbf{p}, \Lambda\right\rangle e^{-\frac{\sigma^{2}}{2} \Delta^{2}} e^{-i \Delta \cdot \mathbf{x}} .
$$

The energy factors $E_{p}=\sqrt{M^{2}+\left(\mathbf{P}-\frac{1}{2} \boldsymbol{\Delta}\right)^{2}}$ and $E_{p}^{\prime}=$ $\sqrt{M^{2}+\left(\mathbf{P}+\frac{1}{2} \boldsymbol{\Delta}\right)^{2}}$ do not factorize in their $\mathbf{P}$ and $\boldsymbol{\Delta}$ dependence. Factorizing the integrand into a $\mathbf{P}$-dependent piece and a $\Delta$-dependent piece would require setting $\boldsymbol{\Delta}=0$, which can be achieved by integrating out $\mathbf{x}$. However, this would preclude obtaining a density.

As previously found in Refs. [35,36], Eq. (12) tells us that dependence on the hadron's wave function cannot be removed or even factored out of the spatial density when using fixed instant form time. Moreover, the expression obtained in Eq. (12) does not resemble the conventional Breit frame Fourier transform at all. One would need to eliminate the $\mathbf{P}$ integral while setting $\mathbf{P}=0$ in order to obtain the Breit frame transform, but such a procedure is incompatible with the spatially localized state that was used. States localized in momentum space-i.e., plane waves - would need to be used to set $\mathbf{P}=0$, but as found in Ref. [35], such a state produces singular densities with infinite radii since the state is completely delocalized.

Therefore, the Breit frame Fourier transform does not give a spatial density. On the other hand, Eq. (12) does give a valid spatial density, but this density does not correspond strictly to the internal structure of the hadron. Wave function spread will always be present in any density defined at fixed $x^{0}$. It is thus preferable to use the light front density of Eq. (7) to describe the hadron structure, since wave function spread can be factored out-and in some cases eliminated entirely.

\section{THE LIGHT FRONT STRESS TENSOR}

Identifying classical mechanical concepts such as pressure and shear forces in inherently quantum mechanical systems such as hadrons is difficult. In practice, "pressure" is identified within hadrons by comparing matrix elements of the EMT for hadron states to a continuum EMT. The matrix element is typically evaluated using plane wave states in the Breit frame, with $\mathbf{P}=0$, so that the stress tensor-which is identified with the spatial components of the EMT-does not depend on the bulk velocity of the hadron.

However, we have established in Sec. II that there is no connection between Breit frame matrix elements and actual spatial densities. Moreover, a properly defined spatial density involves an integral over all values of the transverse momentum $\mathbf{P}_{\perp}$, meaning that the stress tensor contains contributions from the bulk flow of energy and momentum, rather than just forces internal to the hadron. It is necessary to isolate the part of the stress tensor corresponding strictly to internal forces, which we call the "pure stress tensor." We shall proceed to consider how this can be done.

Since the classical EMT is symmetric, we use the symmetric, Belinfante-improved EMT [41] on the field theoretic side of the comparison.

\section{A. Continuum EMT on the light front}

Since spatial densities encoding the internal structure of hadrons can only be defined at equal light front time, with $x^{-}$integrated out, we shall begin by considering the general properties of a classical EMT under these conditions. The Poincaré group has a Galilean subgroup [42-44] that leaves the foliation of spacetime into $x^{+}$slices invariant. Besides the Hamiltonian $P^{-}$, which generates $x^{+}$translations, the remaining generators of the subgroup leave $x^{+}=$fixed invariant. Moreover, transformations in this Galilean subgroup have the special property that the + and transverse components of transformed tensors do not depend on the - components in the original frame.

It is therefore prudent to proceed considering only the $+, 1,2$ components of the EMT and of other tensors. We thus proceed with the inherently $(2+1)$-dimensional quantity:

$T_{\mathrm{LF}}^{\mu \nu}\left(x^{+}, \mathbf{x}_{\perp}\right)=\int \mathrm{d} x^{-} T^{\mu \nu}\left(x^{+}, x^{-}, \mathbf{x}_{\perp}\right), \quad: \mu, \nu=+, 1,2$.

The EMT is made up of two pieces: a flow tensor $V_{\mathrm{LF}}^{\mu \nu}\left(x^{+}, \mathbf{x}_{\perp}\right)$ that encodes the local motion of the continuum material, and the pure stress tensor $S_{\mathrm{LF}}^{\mu \nu}\left(x^{+}, \mathbf{x}_{\perp}\right)$ that encodes mechanical forces,

$$
T_{\mathrm{LF}}^{\mu \nu}\left(x^{+}, \mathbf{x}_{\perp}\right)=V_{\mathrm{LF}}^{\mu \nu}\left(x^{+}, \mathbf{x}_{\perp}\right)+S_{\mathrm{LF}}^{\mu \nu}\left(x^{+}, \mathbf{x}_{\perp}\right) .
$$

The pure stress tensor evaluated at $\mathbf{x}_{\perp}$ is what the EMT evaluates to in a frame that is comoving with the material at $\mathbf{x}_{\perp}$ [45].

To better understand the flow and pure stress tensors, let us first consider a small element of material at transverse rest. $^{1}$ By Noether's theorem, the EMT components

\footnotetext{
${ }^{1}$ By "transverse rest," we mean that once $x^{-}$is integrated out, the net momentum in the transverse plane is zero.
} 
$T_{\mathrm{LF}}^{+\nu}\left(x^{+}, \mathbf{x}_{\perp}\right)$ encode the densities of the momentum components $P^{\nu}$, which for the material at transverse rest gives $T_{\mathrm{LF}, \text { rest }}^{+i}\left(x^{+}, \mathbf{x}_{\perp}\right)=0$. On the other hand, the light front momentum $\left(P^{+}\right)$density is given by

$$
\varepsilon\left(x^{+}, \mathbf{x}_{\perp}\right)=T_{\mathrm{LF}, \text { rest }}^{++}\left(x^{+}, \mathbf{x}_{\perp}\right) .
$$

In this case, the flow tensor is defined by

$$
V_{\mathrm{LF}, \mathrm{rest}}^{\mu \nu}\left(x^{+}, \mathbf{x}_{\perp}\right)=\left[\begin{array}{ccc}
\varepsilon\left(x^{+}, \mathbf{x}_{\perp}\right) & 0 & 0 \\
0 & 0 & 0 \\
0 & 0 & 0
\end{array}\right] .
$$

The pure stress tensor $S_{\mathrm{LF}}^{\mu \nu}\left(x^{+}, \mathbf{x}_{\perp}\right)$ is defined through the remaining spatial components of $T_{\mathrm{LF}}^{\mu \nu}\left(x^{+}, \mathbf{x}_{\perp}\right)$ and can be written out in terms of components as

$$
S_{\mathrm{LF}, \text { rest }}^{\mu \nu}\left(x^{+}, \mathbf{x}_{\perp}\right)=\left[\begin{array}{ccc}
0 & 0 & 0 \\
0 & S_{11}\left(x^{+}, \mathbf{x}_{\perp}\right) & S_{12}\left(x^{+}, \mathbf{x}_{\perp}\right) \\
0 & S_{21}\left(x^{+}, \mathbf{x}_{\perp}\right) & S_{22}\left(x^{+}, \mathbf{x}_{\perp}\right)
\end{array}\right] .
$$

The pure stress tensor has only spatial components in the transverse rest frame of the material element, and under transformations within the light front Galilean subgroupsuch as transverse boosts - this property remains invariant. Thus $S^{++}=S^{+i}=S^{i+}=0$ is true in all frames.

On the other hand, $V_{\mathrm{LF}}^{\mu \nu}\left(x^{+}, \mathbf{x}_{\perp}\right)$ is not invariant under transverse boosts, but instead has the generic form

$$
V_{\mathrm{LF}}^{\mu \nu}\left(x^{+}, \mathbf{x}_{\perp}\right)=u^{\mu} u^{\nu} \varepsilon\left(x^{+}, \mathbf{x}_{\perp}\right),
$$

where $u^{\mu}$ is the light front velocity of the material element, with $u^{+}=1$ and $P^{+} \mathbf{u}_{\perp}=\mathbf{p}_{\perp}$.

A general continuum material consists of many small elements that may be in motion relative to each other. The general form of the continuum EMT on the light front is thus obtained by making the light front velocity a function of space and time:

$$
\begin{aligned}
T_{\mathrm{LF}}^{\mu \nu}\left(x^{+}, \mathbf{x}_{\perp}\right)= & u^{\mu}\left(x^{+}, \mathbf{x}_{\perp}\right) u^{\nu}\left(x^{+}, \mathbf{x}_{\perp}\right) \varepsilon\left(x^{+}, \mathbf{x}_{\perp}\right) \\
& +S_{\mathrm{LF}}^{\mu \nu}\left(x^{+}, \mathbf{x}_{\perp}\right) .
\end{aligned}
$$

It is worth noting that rotational motion of the material can also be encoded through the velocity field $u^{\mu}\left(x^{+}, \mathbf{x}_{\perp}\right)$. However, since this is a classical description, intrinsic quark spin-which, in quantum field theories, is encoded in the antisymmetric contribution to the EMT [9]—cannot be accommodated by Eq. (19).

We shall now proceed to consider properties of $T_{\mathrm{LF}}^{\mu \nu}\left(x^{+}, \mathbf{x}_{\perp}\right), V_{\mathrm{LF}}^{\mu \nu}\left(x^{+}, \mathbf{x}_{\perp}\right)$, and $S_{\mathrm{LF}}^{\mu \nu}\left(x^{+}, \mathbf{x}_{\perp}\right)$. First, the unintegrated EMT obeys a continuity equation,

$$
\partial_{\mu} T^{\mu \nu}(x)=0,
$$

as a consequence of Noether's theorem. If the EMT vanishes at spatial infinity, then the $x^{-}$-integrated EMT also obeys a continuity equation,

$$
\partial_{\mu} T_{\mathrm{LF}}^{\mu \nu}\left(x^{+}, \mathbf{x}_{\perp}\right)=0 .
$$

The pure stress tensors are subject to Cauchy's first law of mechanics [46],

$$
\nabla_{i} S_{\mathrm{LF}}^{i j}\left(x^{+}, \mathbf{x}_{\perp}\right)=-\mathbf{F}_{\perp}^{j}\left(x^{+}, \mathbf{x}_{\perp}\right),
$$

which can be seen to follow from Eq. (21) with the definition

$$
\mathbf{F}_{\perp}\left(x^{+}, \mathbf{x}_{\perp}\right)=\partial_{+}\left[\varepsilon\left(x^{+}, \mathbf{x}_{\perp}\right) \mathbf{u}_{\perp}\left(x^{+}, \mathbf{x}_{\perp}\right)\right],
$$

which is effectively a statement of Newton's second law: a net force produces a change in momentum with time. Thus, $\nabla_{i} S_{\mathrm{LF}}^{i j}\left(x^{+}, \mathbf{x}_{\perp}\right)$ gives the local force acting on elements of the material.

For a system in equilibrium - such as an isolated hadron-the net force at all locations should be zero. ${ }^{2}$ Since $S^{+\nu}=0$, the equilibrium condition can also be written as

$$
\partial_{\mu} S_{\mathrm{LF}}^{\mu \nu}\left(x^{+}, \mathbf{x}_{\perp}\right)=0,
$$

which when combined with the continuity equation for the EMT, also gives

$$
\partial_{\mu} V_{\mathrm{LF}}^{\mu \nu}\left(x^{+}, \mathbf{x}_{\perp}\right)=0 .
$$

This means that, besides the EMT as a whole, the flow tensor and pure stress tensor are separately conserved quantities in an equilibrium system.

\section{B. Gravitational form factors}

When using inherently classical formulas such as Eq. (19) in a quantum mechanical context, the quantities involved should be understood as expectation values using physical states. As found in Sec. II, these expectation values can be expressed as Fourier transforms of matrix elements $\left\langle p^{\prime}, \lambda|\hat{\mathcal{O}}(x)| p, \lambda\right\rangle$ between plane wave states.

The matrix elements $\left\langle p^{\prime}, \lambda\left|T^{\mu \nu}(0)\right| p, \lambda\right\rangle$ in particular define gravitational form factors. For spin-zero hadrons, the standard decomposition is [22]

$$
\left\langle p^{\prime}\left|T^{\mu \nu}(0)\right| p\right\rangle=2 P^{\mu} P^{\nu} A(t)+\frac{\Delta^{\mu} \Delta^{\nu}-\Delta^{2} g^{\mu \nu}}{2} D(t),
$$

\footnotetext{
${ }^{2}$ The force on quarks and the force on gluons may each be nonzero, but need to sum to zero.
} 
where $t=\Delta^{2}$ is the Lorentz-invariant squared momentum transfer; while for spin-half particles

$$
\left\langle p^{\prime}, \lambda\left|T^{\mu \nu}(0)\right| p, \lambda\right\rangle=\bar{u}\left(p^{\prime}, \lambda\right)\left\{\frac{P^{\mu} P^{\nu}}{M} A(t)+\frac{\Delta^{\mu} \Delta^{\nu}-\Delta^{2} g^{\mu \nu}}{4 M} D(t)+\frac{i P^{\{\mu} \sigma^{\nu\} \rho} \Delta_{\rho}}{2 M} J(t)\right\} u(p, \lambda),
$$

where the curly brackets \{\} signify symmetrization over the indices between them (without a factor $1 / 2$ ). Using the Kogut-Soper spinors [47] and $\Delta^{+}=0^{3}$ (as happens to be the case when evaluating spatial densities), we find through explicit evaluation that the $+, 1,2$ components of the spinhalf matrix element can be rewritten:

$$
\begin{aligned}
\left\langle p^{\prime}, \lambda\left|T^{\mu \nu}\left(x^{-}\right)\right| p, \lambda\right\rangle= & 2 P^{\mu} P^{\nu} A(t)+\frac{\Delta^{\mu} \Delta^{\nu}-\Delta^{2} g^{\mu \nu}}{2} D(t) \\
& -i \lambda P^{\{\mu} \epsilon^{\nu\} \rho+} \Delta_{\rho} J(t),
\end{aligned}
$$

where the Levi-Civita symbol should be understood as a three-dimensional quantity with $\epsilon^{+12}=1$, and which we emphasize is true only at $\Delta^{+}=0$.

A few pertinent properties of the flow and pure stress tensors allow us to identify terms in Eqs. (26) and (28) with $V_{\mathrm{LF}}^{\mu \nu}(0)$ and $S_{\mathrm{LF}}^{\mu \nu}(0)$. First, since the hadron is in equilibrium, the flow and pure stress tensors are separately conserved, meaning the Lorentz structures associated with each should contract with $\Delta_{\mu}$ to zero. The Lorentz structures accompanying $A(t), D(t)$, and $J(t)$ each separately satisfy this constraint already. In addition, the facts that $S^{+i}=S^{i+}=$ $S^{++}=0$ and that $V^{i j}$ should vanish for the transverse rest state $\mathbf{P}_{\perp}=0$ allow us to uniquely identify

$$
\begin{aligned}
& \left\langle p^{\prime}, \lambda\left|V_{\mathrm{LF}}^{\mu \nu}(0)\right| p, \lambda\right\rangle \\
& \quad=(2 \pi) \delta\left(\Delta^{+}\right)\left\{2 P^{\mu} P^{\nu} A(t)-i \lambda P^{\{\mu} \epsilon^{\nu\} \rho+} \Delta_{\rho} J(t)\right\}, \\
& \left\langle p^{\prime}, \lambda\left|S_{\mathrm{LF}}^{\mu \nu}(0)\right| p, \lambda\right\rangle=(2 \pi) \delta\left(\Delta^{+}\right)\left(\frac{\Delta^{\mu} \Delta^{\nu}-\Delta^{2} g^{\mu \nu}}{2}\right) D(t),
\end{aligned}
$$

where the $J(t)$ term is absent for spin-zero hadrons. [Note that $V_{\mathrm{LF}}^{\mu \nu}(0)$ and $S_{\mathrm{LF}}^{\mu \nu}(0)$ by definition already include an integral over $x^{-}$, which is why the factors of $\delta\left(\Delta^{+}\right)$are present.]

As explained in the discussion surrounding Eq. (7), one cannot obtain an intrinsic spatial density (i.e., a density that is finite for spatially localized states) from a matrix element that involves factors of $\mathbf{P}_{\perp}^{i}$. The pure stress tensor is an

\footnotetext{
${ }^{3}$ Kogut-Soper spinors give the same results for matrix elements as Brodsky-Lepage spinors [48]. Table II of Ref. [48] contains various matrix elements of these spinors. Note that these matrix elements may be different for spinors with canonical polarization, but light front helicity spinors such as in Refs. [47,48] are more natural to use in the light front formalism.
}

inherently good quantity for defining spatial densities, but of the flow tensor, only $V_{\mathrm{LF}}^{++}=T_{\mathrm{LF}}^{++}$gives a good spatial density. This, of course, means that the spatial components of the EMT-which constitute the entire stress tensor, as conventionally defined (see Refs. [45,46,49])—do not constitute a good intrinsic spatial density, but that the pure stress tensor-which corresponds to how the stress tensor is seen by a comoving observer at each point in the material, and which corresponds to the $D(t)$ form factor-does constitute a good spatial density.

\section{SPATIAL DENSITIES OF THE EMT}

As we have seen in Sec. II, it is only possible to define spatial densities that encode the internal structure of hadrons within the light front formalism. Moreover, such densities can only be defined for operators whose matrix elements between plane wave states do not depend on the average transverse momentum between the states. When these conditions are met, the spatial density is given by Eq. (7).

\section{A. Light front momentum density}

It was found in Sec. III that $T^{++}(x)$ is the only component of the EMT that defines a good spatial density. Using Eq. (7), along with either of the form factor decompositions in Eqs. (26) and (28) gives

$$
\begin{aligned}
\varepsilon\left(\mathbf{x}_{\perp}\right) & \equiv \int \mathrm{d} x^{-}\left\langle\Psi\left|T^{++}(x)\right| \Psi\right\rangle \\
& =P^{+} \int \frac{\mathrm{d}^{2} \boldsymbol{\Delta}_{\perp}}{(2 \pi)^{2}} A(t) e^{-i \Delta_{\perp} \cdot \mathbf{x}_{\perp}} e^{-\frac{\sigma^{2}}{2} \Delta_{\perp}^{2}} .
\end{aligned}
$$

This is the light front momentum density for the + component.

As a momentum density, the integral of $\varepsilon\left(\mathbf{x}_{\perp}\right)$ over all space should give the actual value of the momentum $P^{+}$. Since

$$
\int \mathrm{d}^{2} \mathbf{x}_{\perp} \varepsilon\left(\mathbf{x}_{\perp}\right)=P^{+} A(0)
$$

this imposes the well-known momentum sum rule $A(0)=1$.

The $\mathbf{x}_{\perp}^{2}$-weighted integral of $\varepsilon\left(\mathbf{x}_{\perp}\right)$, when the net momentum $P^{+}$is divided out, defines the square of a light front momentum radius: 


$$
\left\langle x_{\perp}^{2}\right\rangle_{\mathrm{mom}} \equiv \frac{1}{P^{+}} \int \mathrm{d}^{2} \mathbf{x}_{\perp} \mathbf{x}_{\perp}^{2} \varepsilon\left(\mathbf{x}_{\perp}\right)=\left.4 \frac{\mathrm{d} A(t)}{\mathrm{d} t}\right|_{t=0} .
$$

Thus, the light front formalism provides a physical interpretation for the slope of $A(t)$. It is worth remarking that the pion "mass radius" extracted from Ref. [18] is actually $\sqrt{3 / 2}$ times the $P^{+}$radius.

Although it describes a different quantity - energy rather than momentum - the most conceptually adjacent concept provided by the Breit frame formalism is its energy radius $[22,26]$ :

$\left\langle r^{2}\right\rangle_{\text {Breit }}=\left.6 \frac{\mathrm{d} A(t)}{\mathrm{d} t}\right|_{t=0}-\frac{3}{4 M^{2}}\{A(0)-2 J(0)+2 D(0)\}$,

where $J(0)=0$ for spin-zero hadrons and $J(0)=\frac{1}{2}$ for spin-half hadrons. As a theoretical quantity for understanding hadron structure, the light front $P^{+}$radius has several virtues over the Breit frame energy radius. The most obvious of these virtues should be that the light front momentum radius is the radius of an actual spatial density. However, there are several other peculiarities exhibited by the Breit frame radius.

For a spin-zero point particle, one has $A(0)=1$ and $D(0)=-1$ [50]. The Breit frame energy radius does not vanish, but is instead given by $\left\langle r^{2}\right\rangle_{\text {Breit }}=\frac{3}{4 M^{2}}$, while the light front momentum radius vanishes as expected. Additionally, for massless hadrons with either spin zero or spin half, the Breit frame radius is infinite, while the light front momentum radius remains finite.

Another minor benefit of the light front momentum radius is that it is simply given by (a number times) the slope of the form factor $A(t)$.

\section{B. Light front pure stress tensor}

As seen in Sec. III, the spatial components of the EMT do not define a good spatial density, but the pure stress tensor does define a good density. In particular, using Eq. (29) in Eq. (7) gives

$$
\begin{aligned}
S^{i j}\left(\mathbf{x}_{\perp}\right)= & \frac{1}{4 P^{+}} \int \frac{\mathrm{d}^{2} \boldsymbol{\Delta}_{\perp}}{(2 \pi)^{2}}\left(\boldsymbol{\Delta}_{\perp}^{i} \Delta_{\perp}^{j}-\Delta_{\perp}^{2} \delta^{i j}\right) \\
& \times D(t) e^{-i \Delta_{\perp} \cdot \mathbf{x}_{\perp}} e^{-\frac{\sigma^{2}}{2} \Delta_{\perp}^{2}} .
\end{aligned}
$$

The pure stress tensor, notably, vanishes in the infinite momentum limit (i.e., as $P^{+} \rightarrow \infty$ ), but in any physically realistic frame (in which $P^{+}$is finite), the pure stress tensor is finite. Because of the factor $\frac{1}{P^{+}}$, however, the pure stress tensor is not invariant under longitudinal boosts, and it is additionally not independent of state preparation. Accordingly, any "forces" encoded in the pure stress tensor are also frame- and state-dependent. On the other hand, by multiplying by $P^{+}$, one can obtain a frame- and stateindependent quantity:

$$
\begin{aligned}
\tilde{S}^{i j}\left(\mathbf{x}_{\perp}\right)= & P^{+} S^{i j}\left(\mathbf{x}_{\perp}\right)=\frac{1}{4} \int \frac{\mathrm{d}^{2} \boldsymbol{\Delta}_{\perp}}{(2 \pi)^{2}}\left(\Delta_{\perp}^{i} \Delta_{\perp}^{j}-\Delta_{\perp}^{2} \delta^{i j}\right) \\
& \times D(t) e^{-i \Delta_{\perp} \cdot \mathbf{x}_{\perp}} e^{-\frac{\sigma^{2}}{2} \Delta_{\perp}^{2}} .
\end{aligned}
$$

The physical meaning of this expression is explored below through examples.

The pure stress tensor $S^{i j}\left(\mathbf{x}_{\perp}\right)$ is isotropic for spin-zero and spin-half particles, as can be seen from examining Eq. (34). This means that it can be parametrized by two independent functions of $x_{\perp}^{2}$ :

$$
S^{i j}\left(\mathbf{x}_{\perp}\right)=\delta^{i j} p\left(x_{\perp}^{2}\right)+\left(\frac{x_{\perp}^{i} x_{\perp}^{j}}{x_{\perp}^{2}}-\frac{1}{2} \delta^{i j}\right) s\left(x_{\perp}^{2}\right) .
$$

Here, $p\left(x_{\perp}^{2}\right)$ is the light front static pressure, and the remaining components-parametrized by the function $s\left(x_{\perp}^{2}\right)$ - give the stress deviator tensor [46], whose components are shear stresses $[45,46]$.

A few conceptual remarks are in order regarding the physical meaning of the pressure and shear forces in the light front stress tensor. With $x^{-}$integrated out, the description given by $S^{i j}\left(\mathbf{x}_{\perp}\right)$ is inherently $(2+1)$ dimensional. Surfaces in two-dimensional space are onedimensional, and accordingly the light front pressure has units of force/length rather than force/area [as can be confirmed through a unit analysis on Eq. (34)].

\section{Properties of pressure and shear functions}

Although the Breit frame Fourier transform does not actually give a spatial density, a significant amount of work has been done using the Breit frame EMT. It is thus instructive to construct analogies on the light front to results that have already been obtained in literature using the Breit frame. Reference [22] is especially helpful to compare to.

To begin, in analogy to Eq. (23) of Ref. [22], we find that the pressure and shear functions can be written as

$$
\begin{aligned}
\tilde{D}\left(x_{\perp}\right) & =\frac{1}{4 P^{+}} \int \frac{\mathrm{d}^{2} \boldsymbol{\Delta}_{\perp}}{(2 \pi)^{2}} D(t) e^{-i \Delta_{\perp} \cdot \mathrm{x}_{\perp},} \\
p\left(x_{\perp}\right) & =\frac{1}{2 x_{\perp}} \frac{\mathrm{d}}{\mathrm{d} x_{\perp}} \tilde{D}\left(x_{\perp}\right)+\frac{1}{2} \frac{\mathrm{d}^{2}}{\mathrm{~d} x_{\perp}^{2}} \tilde{D}\left(x_{\perp}\right) \\
& =\frac{1}{2 x_{\perp}} \frac{\mathrm{d}}{\mathrm{d} x_{\perp}}\left[x_{\perp} \frac{\mathrm{d}}{\mathrm{d} x_{\perp}} \tilde{D}\left(x_{\perp}\right)\right], \\
s\left(x_{\perp}\right) & =\frac{1}{x_{\perp}} \frac{\mathrm{d}}{\mathrm{d} x_{\perp}} \tilde{D}\left(x_{\perp}\right)-\frac{\mathrm{d}^{2}}{\mathrm{~d} x_{\perp}^{2}} \tilde{D}\left(x_{\perp}\right) \\
& =-x_{\perp} \frac{\mathrm{d}}{\mathrm{d} x_{\perp}}\left[\frac{1}{x_{\perp}} \frac{\mathrm{d}}{\mathrm{d} x_{\perp}} \tilde{D}\left(x_{\perp}\right)\right] .
\end{aligned}
$$


The pressure in particular has another simple expression:

$$
p\left(x_{\perp}\right)=\frac{1}{2} \delta_{i j} S^{i j}\left(\mathbf{x}_{\perp}\right)=-\frac{1}{8 P^{+}} \int \frac{\mathrm{d}^{2} \boldsymbol{\Delta}_{\perp}}{(2 \pi)^{2}} \boldsymbol{\Delta}_{\perp}^{2} D(t) e^{-i \Delta_{\perp} \cdot \mathbf{x}_{\perp}} .
$$

From this expression, it is clear that the von Laue stability condition $[22,51]$

$$
\int \mathrm{d}^{2} \mathbf{x}_{\perp} p\left(\mathbf{x}_{\perp}\right)=0
$$

is automatically satisfied for any hadron.

Equations (37) show that $p\left(x_{\perp}\right)$ and $s\left(x_{\perp}\right)$ are not independent functions. In analogy to the discussion around Eq. (30) of Ref. [22], the nonindependence of these functions can also be demonstrated through the equilibrium condition $\nabla_{i} S_{\mathrm{LF}}^{i j}\left(\mathbf{x}_{\perp}\right)=0$. This condition entails

$$
p^{\prime}\left(x_{\perp}\right)+\frac{1}{2} s^{\prime}\left(x_{\perp}\right)+\frac{1}{x_{\perp}} s\left(x_{\perp}\right)=0
$$

which is the 2D light front analogy to Eq. (30) of Ref. [22]. Here we use $p^{\prime}\left(x_{\perp}\right)$ to denote differentiation with respect to $x_{\perp}=\left|\mathbf{x}_{\perp}\right|$, i.e.,

$$
p^{\prime}\left(x_{\perp}\right)=\frac{\mathrm{d} p\left(\left|\mathbf{x}_{\perp}\right|\right)}{\mathrm{d}\left|\mathbf{x}_{\perp}\right|} .
$$

Expressions for the light front pressure and shear densities-as well as the normal and tangential force distributions-were previously found in Sec. IV of Ref. [32]. Equations (110) and (111) of Ref. [32] in particular agree with Eqs. (37) of the current work, up to the minor difference that $\frac{M}{P^{+}}$was factored out of each of these densities in Ref. [32]. This work and Ref. [32] arrive at the same expressions for different reasons, however. The stress tensor contains a velocity flow piece with factors of $\mathbf{P}_{\perp}$ that blows up for spatially localized $\left(\mathbf{R}_{\perp}=0\right)$ states. In this work, we identified pressure and shear by looking at the "pure stress tensor," in which the velocity flow piece is removed. In Ref. [32], the EMT density was defined in phase space using a Wigner function formalism, and the condition $\mathbf{P}_{\perp}=0$ was imposed throughout Sec. IV. This removes exactly the same terms from the stress tensor as we removed from the pure stress tensor, and since the remaining terms have no $\mathbf{P}_{\perp}$ dependence, the results for the densities are the same. We remark, however, that integration over all $\mathbf{P}_{\perp}$ is needed to obtain a physical density from a Wigner density, and the condition $\mathbf{P}_{\perp}=0$ cannot be imposed while integrating over $\mathbf{P}_{\perp}$.

\section{Stability conditions and mechanical radius}

As described above, the von Laue condition-that the integral of pressure over all space is zero-is automatically satisfied for any hadron. Another important stability condition is the Polyakov-Schweitzer negativity condition [22], which stipulates that the $x_{\perp}^{2}$-weighted moment of pressure should be negative for any mechanically stable system. We find the relevant moment to be

$$
\int \mathrm{d}^{2} \mathbf{x}_{\perp} \mathbf{x}_{\perp}^{2} p\left(\mathbf{x}_{\perp}\right)=\frac{1}{2 P^{+}} D(0),
$$

meaning the Polyakov-Schweitzer condition can be written as

$$
D(0) \leq 0
$$

This is identical to Eq. (40) of Ref. [22] [up to the possibility that $D(0)=0$, which is satisfied, for instance, by free fermions]. Unlike the von Laue condition, satisfying the negativity condition is not trivial, and the requirement that $D(0) \leq 0$ can provide a useful sanity check for model calculations of hadron structure.

A more general (nontrivial) stability condition can be derived, following the discussion around Eq. (39) of Ref. [22]. The effective normal force density on a 1D surface-with units force/length-is given by $S^{i j}\left(\mathbf{x}_{\perp}\right) \hat{n}_{i}$, where $\hat{n}$ is a unit vector normal to the surface. ${ }^{4}$ For a system centered at the origin, the requirement that normal forces be directed strictly outwards - a stability condition protecting against collapserequires that $S^{i j}\left(\mathbf{x}_{\perp}\right) \mathbf{x}_{\perp}^{i} \geq=0$, which imposes

$$
p\left(\mathbf{x}_{\perp}\right)+\frac{1}{2} s\left(\mathbf{x}_{\perp}\right) \geq 0,
$$

which can be seen as a two-dimensional light front analog of Eq. (39) from Ref. [22], and which was also previously found in Eq. (128) of Ref. [32]. In terms of the function $\tilde{D}\left(x_{\perp}\right)$ defined in Eq. (37), this stability condition can be written as

$$
\frac{1}{x_{\perp}} \tilde{D}^{\prime}\left(x_{\perp}\right) \geq 0
$$

which entails

$$
\int \frac{\mathrm{d}^{2} \boldsymbol{\Delta}_{\perp}}{(2 \pi)^{2}}\left\{D(t)+t \frac{\mathrm{d} D(t)}{\mathrm{d} t}\right\} e^{-i \Delta_{\perp} \cdot \mathbf{x}_{\perp}} \leq 0 .
$$

By integrating this condition over all space, one obtains Eq. (42) as a special case. Equation (45) is, however, a stricter condition.

\footnotetext{
${ }^{4}$ It is, in fact, the pure stress tensor-the local value of the stress tensor as measured by a comoving observer-that corresponds to the normal force density. See Chapter 12 of Ref. [45].
} 
As noted in Ref. [22], the normal force being positive (directed outwards) means it can be used to define a mean squared radius, which is called the mechanical radius in Ref. [22]. Within the light front radius, we find the mechanical radius to be

$$
\left\langle x_{\perp}^{2}\right\rangle_{\text {mech }}=\frac{\int \mathrm{d}^{2} \mathbf{x}_{\perp} \mathbf{x}_{\perp}^{2}\left[p\left(\mathbf{x}_{\perp}\right)+\frac{1}{2} s\left(\mathbf{x}_{\perp}\right)\right]}{\int \mathrm{d}^{2} \mathbf{x}_{\perp}\left[p\left(\mathbf{x}_{\perp}\right)+\frac{1}{2} s\left(\mathbf{x}_{\perp}\right)\right]}=\frac{4 D(0)}{\int_{-\infty}^{0} \mathrm{~d} t D(t)},
$$

which is the light front analog of Eq. (41) from Ref. [22].

\section{ILLUSTRATIONS WITH A DIPOLE PARAMETRIZATION}

Several phenomenological parametrizations and model calculations of the gravitational form factors $A(t)$ and $D(t)$ for various mesons and baryons exist in the literature (see Refs. [26-29,52], for instance). Although it is not universal, it is fairly common to use a multipole parametrization to approximate the results of model calculations. The generic multipole parametrizations take the form

$$
\begin{aligned}
& A(t)=\frac{1}{\left(1-t / \Lambda^{2}\right)^{n}}, \\
& D(t)=\frac{D(0)}{\left(1-t / \Lambda^{2}\right)^{n}},
\end{aligned}
$$

where $n$ is the order of the multipole and $\Lambda$ is the multipole mass. $n$ and $\Lambda$ can be different for $A(t)$ and $D(t)$, but to reduce notational clutter we use the same notation for both. We shall proceed to use these standard ansatzes for the gravitational form factors to illustrate the light front densities with the EMT.

\section{A. General formulas using the multipole form}

First, let us consider the light front momentum density, which is given by Eq. (30). Using the multipole parametrization, we obtain

$$
\varepsilon\left(\mathbf{x}_{\perp}\right)=P^{+} \int \frac{\mathrm{d}^{2} \boldsymbol{\Delta}_{\perp}}{(2 \pi)^{2}} \frac{e^{-i \Delta_{\perp} \cdot \mathbf{x}_{\perp}}}{\left(1+\boldsymbol{\Delta}_{\perp}^{2} / \Lambda^{2}\right)^{n}} .
$$

This integral can be approached by breaking $\boldsymbol{\Delta}_{\perp}$ into a component parallel to $\mathbf{x}_{\perp}$ and an orthogonal component, and integrating out the latter. This gives

$\varepsilon\left(\mathbf{x}_{\perp}\right)=P^{+} \frac{\Lambda^{2 n}}{4 \pi^{2}} \frac{\sqrt{\pi} \Gamma\left(n-\frac{1}{2}\right)}{\Gamma(n)} \int_{-\infty}^{\infty} \mathrm{d} k \frac{e^{-i k x_{\perp}}}{\left(\Lambda^{2}+k^{2}\right)^{n-1 / 2}}$.

Comparing to Basset's integral [53] gives

$$
\varepsilon\left(\mathbf{x}_{\perp}\right)=P^{+} \Lambda^{2} \frac{\left(\Lambda x_{\perp}\right)^{n-1}}{2^{n} \pi \Gamma(n)} K_{n-1}\left(\Lambda x_{\perp}\right)
$$

where $K_{n}(x)$ is a modified Bessel function of the second kind [53].

For the pure stress tensor, and the associated pressure and shear functions, it is most straightforward to use $\tilde{D}\left(x_{\perp}\right)$ as defined in Eqs. (37). In the multipole parametrization

$$
\tilde{D}\left(x_{\perp}\right)=\frac{D(0)}{4 P^{+}} \int \frac{\mathrm{d}^{2} \boldsymbol{\Delta}_{\perp}}{(2 \pi)^{2}} \frac{e^{-i \Delta_{\perp} \cdot \mathbf{x}_{\perp}}}{\left(1+\boldsymbol{\Delta}_{\perp}^{2} / \Lambda^{2}\right)^{n}},
$$

which can be evaluated as

$$
\tilde{D}\left(x_{\perp}\right)=\frac{D(0) \Lambda^{2}}{4 P^{+}} \frac{\left(\Lambda x_{\perp}\right)^{n-1}}{2^{n} \pi \Gamma(n)} K_{n-1}\left(\Lambda x_{\perp}\right) .
$$

Using Eqs. (37) and recursion relations for the modified Bessel functions [53], the pressure and shear functions are

$$
\begin{aligned}
p\left(x_{\perp}\right)= & \frac{D(0) \Lambda^{4}}{4 P^{+}} \frac{1}{2^{n+1} \pi \Gamma(n)}\left\{\left(\Lambda x_{\perp}\right)^{n-1} K_{n-3}\left(\Lambda x_{\perp}\right)\right. \\
& \left.-2\left(\Lambda x_{\perp}\right)^{n-2} K_{n-2}\left(\Lambda x_{\perp}\right)\right\}
\end{aligned}
$$

$s\left(x_{\perp}\right)=-\frac{D(0) \Lambda^{4}}{4 P^{+}} \frac{1}{2^{n} \pi \Gamma(n)}\left(\Lambda x_{\perp}\right)^{n-1} K_{n-3}\left(\Lambda x_{\perp}\right)$.

Here, in the cases $n=2$ (dipole form) and $n=1$ (monopole form), the modified Bessel functions with negative index are equal to the same function with a positive index. Interestingly, although the pressure can flip signs (and must, in order to satisfy the von Laue condition), the shear function is strictly positive, since $D(0)<0$ and since the modified Bessel functions of the second kind are strictly positive.

The normal force is given by the left-hand side of Eq. (43), and in the dipole parametrization is

$$
\begin{aligned}
F_{n}\left(x_{\perp}\right) & =p\left(x_{\perp}\right)+\frac{1}{2} s\left(x_{\perp}\right) \\
& =-\frac{D(0) \Lambda^{4}}{4 P^{+}} \frac{\left(\Lambda x_{\perp}\right)^{n-2}}{2^{n} \pi \Gamma(n)} K_{n-2}\left(\Lambda x_{\perp}\right) .
\end{aligned}
$$

Since $D(0)<0$ and the modified Bessel function is positive, $F_{n}\left(x_{\perp}\right)>0$, as expected of a system stable against collapse.

Using Eq. (32), the light front momentum radius in the multipole parametrization is

$$
\left\langle x_{\perp}^{2}\right\rangle_{\mathrm{mom}}=\frac{4 n}{\Lambda^{2}} .
$$

On the other hand, the mechanical radius for the multipole form can be found using Eq. (46), giving

$$
\left\langle x_{\perp}^{2}\right\rangle_{\text {mech }}=\frac{4(n-1)}{\Lambda^{2}} .
$$



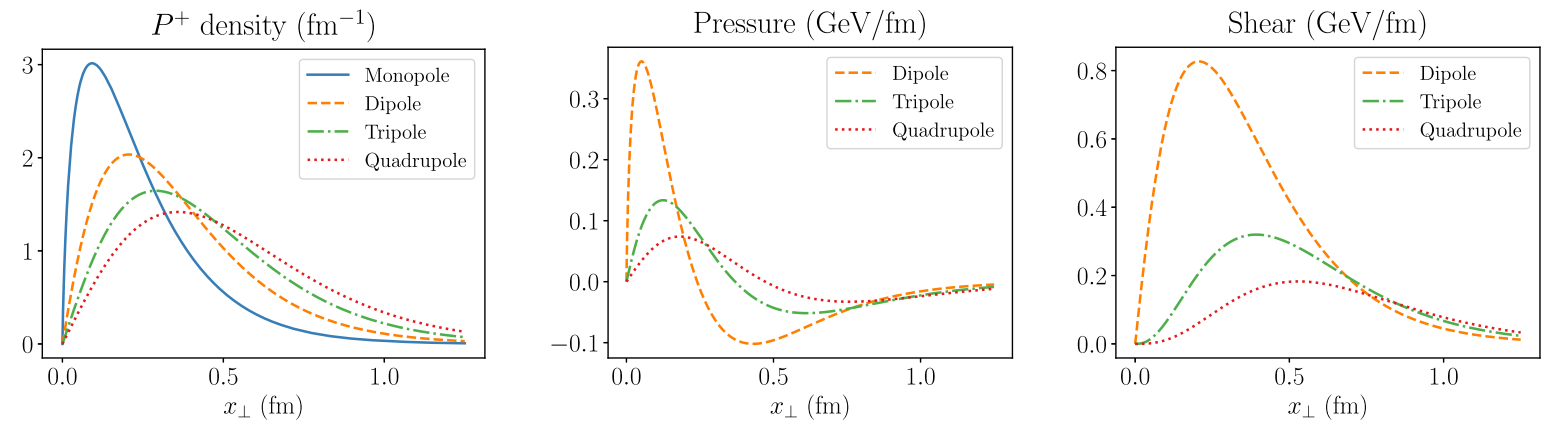

FIG. 1. Light front transverse densities associated with the EMT, for several multipole orders. Each density is weighted by $2 \pi x_{\perp}$. The $P^{+}$density is divided by $P^{+}$, while the pressure and shear are multiplied by $P^{+}$, to remove the $P^{+}$dependence.

\section{B. Singular behavior for specific multipoles}

For $x \sim 0$, the modified Bessel functions of the second kind have the following limited form for positive $n$ [53]:

$$
K_{n}(x) \sim \frac{1}{2} \Gamma(n)\left(\frac{1}{2} x\right)^{-n}
$$

By contrast, $K_{0}(x)$ diverges logarithmically as $x \rightarrow 0$.

When the monopole form $(n=1)$ is used for $A(t)$, the light front momentum density Eq. (50) is logarithmically singular at the origin. This density remaining finite at the origin has been stipulated as a stability condition, for instance, in Ref. [32]. However, a density that is singular at the origin seems to actually occur in QCD, in the case of the pion transverse charge density [54]. It is worth noting that while $\varepsilon\left(x_{\perp}\right)$ is singular in the monopole case at $x_{\perp}=0$, the integral of $\varepsilon\left(x_{\perp}\right)$ over any finite region of space-even one containing the origin-is strictly finite.

Whether a transverse density can be singular at the origin remains an open question. However, lattice data suggest a monopole form for the $A(t)$ form factor of the

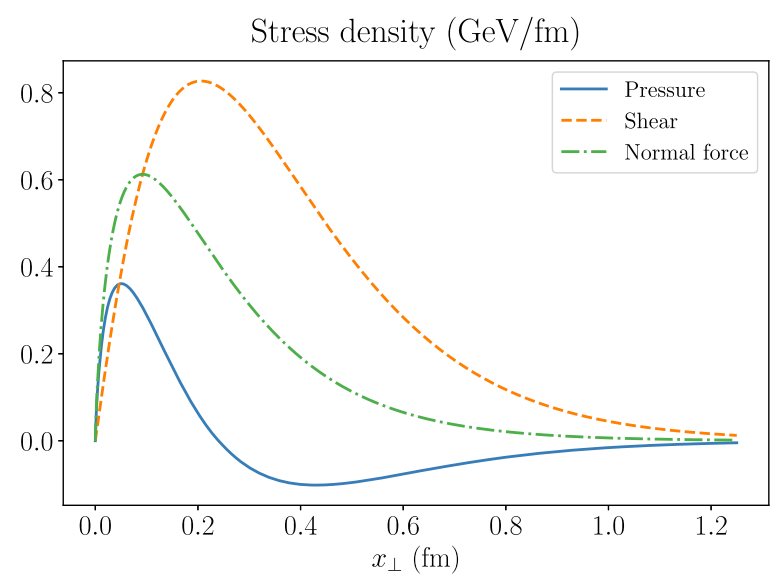

FIG. 2. Light front transverse densities associated with the pure stress tensor using the dipole $(n=2)$ ansatz. Each density is weighted by $2 \pi x_{\perp}$ and multiplied by $P^{+}$to remove the $P^{+}$ dependence. pion [55]. Thus, it seems prudent to not too hastily discard the possibility of transverse densities that are singular at the origin.

As noted in Ref. [32], using the dipole form $(n=2)$ for the $D(t)$ form factor results in a shear function $s\left(x_{\perp}\right)$ that is finite at $x_{\perp}=0$; it also results in a pressure function $p\left(x_{\perp}\right)$ that is singular at the origin. These can be observed by looking at Eqs. (53) and recalling that $K_{-n}(x)=K_{n}(x)$ in these formulas. In particular, $s(0) \neq 0$ would make the offdiagonal components of $S_{\mathrm{LF}}^{i j}(0)$ diverge, as can be seen in Eq. (36).

The requirement that all components of $S_{\mathrm{LF}}^{i j}(0)$ be finite would thus rule out the dipole form for $D(t)$. However, we do not see a fundamental reason why components of the pure stress tensor must be finite at the origin. Indeed, as is the case with $\varepsilon\left(x_{\perp}\right)$ with the monopole form, the integral of $S_{\mathrm{LF}}^{i j}\left(x_{\perp}\right)$ over any region of space-including one that contains the origin-is finite. It seems prudent to not discard the possibility of a dipole form for $D(t)$, though the singular densities this would entail should be kept in mind.

\section{Numerical illustrations}

We will now consider some specific values of the multipole form to illustrate the properties of the light front densities. As mentioned in Ref. [52], the meson dominance hypothesis suggests the presence of an $f_{2}$ pole in the gravitational form factors. We thus use $\Lambda=1.275 \mathrm{GeV}$ [56]. We consider $n=1,2,3,4$ (monopole, dipole, tripole, and quadrupole) forms. Note that Eq. (46) excludes the monopole from consideration for $D(t)$, since it would result in an infinite integral for $F_{n}\left(x_{\perp}\right)$, and thus a zero mechanical radius. For the $D(t)$ form factor specifically, we use $D(0)=-1$ for the purposes of illustration. It is noteworthy that $D(0) \approx-1$ for the pion [22,26,57,58], perhaps making these illustrations most illuminating for the pion specifically.

First, we give numerical values for the light front and mechanical radii in Table I. The monopole form gives a zero mechanical radius because the associated "charge," in 
TABLE I. Values for the light front momentum and mechanical radii using the multipole ansatz in Eq. (47), with $\Lambda=1.275 \mathrm{GeV}$ and $D(0)=-1$, for several orders of multipole.

\begin{tabular}{cccc}
\hline \hline$n$ & $\sqrt{\left\langle x_{\perp}^{2}\right\rangle_{\mathrm{mom}}}$ & $\sqrt{\left\langle x_{\perp}^{2}\right\rangle_{\text {mech }}}$ & $F_{n}(0)$ \\
\hline 1 & $0.310 \mathrm{fm}$ & 0 & $\ldots$ \\
2 & $0.438 \mathrm{fm}$ & $0.219 \mathrm{fm}$ & $\infty$ \\
3 & $0.536 \mathrm{fm}$ & $0.310 \mathrm{fm}$ & $0.338 \mathrm{GeV} / \mathrm{fm}^{2}$ \\
4 & $0.619 \mathrm{fm}$ & $0.379 \mathrm{fm}$ & $0.113 \mathrm{GeV} / \mathrm{fm}^{2}$ \\
\hline \hline
\end{tabular}

the denominator of Eq. (46), is infinite for $n=1$. For the dipole form, the radii are curiously equal; however, it may turn out that $A(t)$ and $D(t)$ are multipoles of different orders, or even that the effective multipole masses are different, so one should not read too much into this coincidence.

The momentum, pressure, and shear densities are plotted in Fig. 1 for several different orders of the multipole ansatz. The $P^{+}$density, with $P^{+}$divided out, integrates to $1-$ a fact that is preserved by a conservation law. The main effect of increasing the multipole order on this density is to make the $P^{+}$density more diffuse in the transverse plane, a fact that is reflected in the increasing momentum radii in Table I. By contrast, rather than merely becoming more diffuse, the pressure and shear drop in magnitude when the multipole order is increased.

The magnitude of forces within the hadron decreasing with multipole order is also reflected in Table I, where the normal force at the center of the hadron decreases with increasing multipole order. Thus, knowing only $D(0)$ is insufficient to know the magnitude of forces (such as pressure) within a hadron; it is necessary to know the full functional form of $D(t)$.

In Fig. 2, we plot the pressure, shear, and normal force for the dipole $(n=2)$ ansatz specifically. In contrast to liquid drop models [22], the shear function is not confined to a narrow region of space and is, in fact, quite broad, exceeding the pressure and the net normal force in magnitude. This finding was also observed for the multipole ansatz in Ref. [32]. On the one hand, the broadness of the shear function suggests that the hadron cannot be seen as having a sharp boundary. On the other hand, the large magnitude of the shear function suggests the hadron is extremely viscous and cannot be interpreted as an ideal fluid. Of course, these qualitative conclusions hold only if the dipole ansatz for $D(t)$ is, in fact, accurate.

\section{CONCLUSIONS AND OUTLOOK}

In this work, we derived the relativistically correct expression for a hadron's transverse rest frame ("pure") stress tensor. The main result is given in Eq. (34), which tells us how the $D$-term $D(t)$ encodes the spatial distribution of forces in the transverse plane at fixed light front time. Since the subgroup that keeps slices of fixed $x^{+}$is Galilean, the pure stress tensor $S^{i j}\left(\mathbf{x}_{\perp}\right)$ has the same formal properties as the classical, nonrelativistic stress tensor, meaning that the classical laws of mechanics (such as Cauchy's laws [46]) can be applied.

Along the way, we obtained general expressions for spatial densities within both the front form and instant form formalisms. We found that in the instant form formalism, one does not obtain a Breit frame Fourier transform. Moreover, we reproduced the result of Ref. [36] that the spatial extent of the hadron's wave function cannot be removed from the density in the instant form formalism.

It is worth stressing that the results we have obtained were derived, with a fundamental field-theoretic quantitythe expectation value of a local operator with a physically realistic state - as the starting point. The spatial densities we have obtained were not postulated nor defined by fiat, and thus their interpretation is in clear and direct correspondence with the actual spatial densities of hadrons.

In this work, we applied the formalism to spin-zero and spin-half hadrons, and we did not explore the spatial distribution of angular momentum. Future work remains to be done on the distribution of angular momentum and spin, as well as the application of this formalism to spin-one particles, which can have quadrupole moments [59], suggesting the stress tensor will not be isotropic.

\section{ACKNOWLEDGMENTS}

The authors thank Julia Yu. Panteleeva for finding and correcting a factor of 2 mistake in the mechanical radius results. This work was supported by the U.S. Department of Energy Office of Science, Office of Nuclear Physics under Award No. DE-FG02-97ER-41014. 
[1] X.-D. Ji, Phys. Rev. Lett. 74, 1071 (1995).

[2] X.-D. Ji, Phys. Rev. D 52, 271 (1995).

[3] C. Lorcé, Eur. Phys. J. C 78, 120 (2018).

[4] Y. Hatta, A. Rajan, and K. Tanaka, J. High Energy Phys. 12 (2018) 008.

[5] S. Rodini, A. Metz, and B. Pasquini, J. High Energy Phys. 09 (2020) 067.

[6] A. Metz, B. Pasquini, and S. Rodini, Phys. Rev. D 102, 114042 (2020).

[7] J. Ashman et al. (European Muon Collaboration), Phys. Lett. B 206, 364 (1988).

[8] X.-D. Ji, Phys. Rev. Lett. 78, 610 (1997).

[9] E. Leader and C. Lorcé, Phys. Rep. 541, 163 (2014).

[10] I. Y. Kobzarev and L. B. Okun, Zh. Eksp. Teor. Fiz. 43, 1904 (1962).

[11] X.-D. Ji, Phys. Rev. D 55, 7114 (1997).

[12] A. V. Radyushkin, Phys. Rev. D 56, 5524 (1997).

[13] B. Kriesten, S. Liuti, L. Calero-Diaz, D. Keller, A. Meyer, G. R. Goldstein, and J. Osvaldo Gonzalez-Hernandez, Phys. Rev. D 101, 054021 (2020).

[14] M. Defurne et al. (Jefferson Lab Hall A Collaboration), Phys. Rev. C 92, 055202 (2015).

[15] H. S. Jo et al. (CLAS Collaboration), Phys. Rev. Lett. 115, 212003 (2015).

[16] M. Hattawy et al. (CLAS12 Run-Group Collaboration), arXiv:1908.00949.

[17] A. Accardi et al., Eur. Phys. J. A 52, 268 (2016).

[18] S. Kumano, Q.-T. Song, and O. V. Teryaev, Phys. Rev. D 97, 014020 (2018).

[19] M. V. Polyakov and C. Weiss, Phys. Rev. D 60, 114017 (1999).

[20] M. V. Polyakov and P. Schweitzer, Proc. Sci., SPIN2018 (2019) 066 [arXiv:1812.06143].

[21] M. V. Polyakov, Phys. Lett. B 555, 57 (2003).

[22] M. V. Polyakov and P. Schweitzer, Int. J. Mod. Phys. A 33, 1830025 (2018).

[23] P. E. Shanahan and W. Detmold, Phys. Rev. Lett. 122, 072003 (2019).

[24] V. D. Burkert, L. Elouadrhiri, and F. X. Girod, Nature (London) 557, 396 (2018).

[25] K. Kumerički, Nature (London) 570, E1 (2019).

[26] A. Freese and I. C. Cloët, Phys. Rev. C 100, 015201 (2019).

[27] I. V. Anikin, Particles 2, 357 (2019).

[28] M. J. Neubelt, A. Sampino, J. Hudson, K. Tezgin, and P. Schweitzer, Phys. Rev. D 101, 034013 (2020).

[29] M. Varma and P. Schweitzer, Phys. Rev. D 102, 014047 (2020).

[30] R. Sachs, Phys. Rev. 126, 2256 (1962).

[31] G. A. Miller, Phys. Rev. C 80, 045210 (2009).
[32] C. Lorcé, H. Moutarde, and A. P. Trawiński, Eur. Phys. J. C 79, 89 (2019).

[33] M. Burkardt, Int. J. Mod. Phys. A 18, 173 (2003).

[34] G. A. Miller, Phys. Rev. Lett. 99, 112001 (2007).

[35] G. A. Miller, Phys. Rev. C 99, 035202 (2019).

[36] R. L. Jaffe, Phys. Rev. D 103, 016017 (2021) .

[37] J. von Neumann, Mathematical Foundations of Quantum Mechanics (Princeton University Press, 1955).

[38] T. D. Newton and E. P. Wigner, Rev. Mod. Phys. 21, 400 (1949).

[39] A. J. Kalnay and B. P. Toledo, Nuovo Cimento 48, 997 (1967).

[40] M. Pavšič, Mod. Phys. Lett. A 33, 1850114 (2018).

[41] F. J. Belinfante, Physica 6, 887 (1939).

[42] P. A. M. Dirac, Rev. Mod. Phys. 21, 392 (1949).

[43] L. Susskind, Phys. Rev. 165, 1535 (1968).

[44] S. J. Brodsky, H.-C. Pauli, and S. S. Pinsky, Phys. Rep. 301, 299 (1998).

[45] A. L. Fetter and J. D. Walecka, Theoretical Mechanics of Particles and Continua, International Series in Pure and Applied Physics (McGraw-Hill, New York, NY, 1980).

[46] F. Irgens, Continuum Mechanics (Springer, Berlin, Heidelberg, 2008).

[47] J. B. Kogut and D. E. Soper, Phys. Rev. D 1, 2901 (1970).

[48] G. P. Lepage and S. J. Brodsky, Phys. Rev. D 22, 2157 (1980).

[49] C. Batchelor and G. Batchelor, An Introduction to Fluid Dynamics, Cambridge Mathematical Library (Cambridge University Press, Cambridge, England, 2000).

[50] J. Hudson and P. Schweitzer, Phys. Rev. D 97, 056003 (2018).

[51] M. Laue, Ann. Phys. (Berlin) 340, 524 (1911).

[52] P. Masjuan, E. Ruiz Arriola, and W. Broniowski, Phys. Rev. D 87, 014005 (2013).

[53] DLMF, in NIST Digital Library of Mathematical Functions, http://dlmf.nist.gov/, Release 1.1.0 of 2020-12-15, edited by F. W. J. Olver, A. B. Olde Daalhuis, D. W. Lozier, B. I. Schneider, R. F. Boisvert, C. W. Clark, B. R. Miller, B. V. Saunders, H. S. Cohl, and M. A. McClain.

[54] G. A. Miller, Phys. Rev. C 79, 055204 (2009).

[55] D. Brommel, Pion structure from the lattice, Ph.D. thesis, Regensburg U., 2007.

[56] P. A. Zyla et al. (Particle Data Group), Prog. Theor. Exp. Phys. (2020), $083 \mathrm{C} 01$.

[57] V. A. Novikov and M. A. Shifman, Z. Phys. C 8, 43 (1981).

[58] M. B. Voloshin and V. I. Zakharov, Phys. Rev. Lett. 45, 688 (1980).

[59] W. Cosyn, S. Cotogno, A. Freese, and C. Lorcé, Eur. Phys. J. C 79, 476 (2019). 